Copyright $(2008$ Institute of Electrical and Electronics Engineers, Inc.

All rights reserved.

Personal use of this material, including one hard copy reproduction, is permitted.

Permission to reprint, republish and/or distribute this material in whole or in part for any other purposes must be obtained from the IEEE.

For information on obtaining permission, send an e-mail message to stds-ipr@ieee.org.

By choosing to view this document, you agree to all provisions of the copyright laws protecting it.

Individual documents posted on this site may carry slightly different copyright restrictions.

For specific document information, check the copyright notice at the beginning of each document. 


\title{
A Novel Classifier Selection Approach for Adaptive Boosting Algorithms
}

\author{
ABM Shawkat Ali and Tony Dobele \\ School of Computing Sciences \\ Central Queensland University \\ Rockhampton, QLD 4702, Australia \\ E-mail: \{s.ali,t.dobele\}@cqu.edu.au
}

\begin{abstract}
Boosting is a general approach for improving classifier performances. In this research we investigated these issues with the latest Boosting algorithm AdaBoostM1. A trial and error classifier feeding with the AdaBoostM1 algorithm is a regular practice for classification tasks in the research community. We provide a novel statistical informationbased rule method for unique classifier selection with the AdaBoostM1 algorithm. The solution also verified a wide range of benchmark classification problems.
\end{abstract}

\section{Introduction}

Boosting is a well established method in the machine learning community for improving the performance of any learning algorithm. The boosting algorithms were first presented by Schapire [1] and Freund [2]. Continuing this research they introduced a new generation of boosting algorithm called Adaptive Boosting (AdaBoost) [3-7]. Schapire and Freund argued that this new boosting algorithm has certain properties which make it more practical and easier to implement than its predecessors [8]. In this paper we investigate this issue within a wide range of classification problems.

During the AdaBoost performance testing we noted that classifier feeding with the AdaBoost algorithm is a trial and error approach. It is a lengthy process to find out a suitable classifier for a specific problem. Our present research provides a statistical informationbased classifier selection for the AdaBoost algorithm. The rest of the paper is organized as follows: first we provide a brief description of boosting, AdaBoostM1 and some popular base classifiers. After that we summarize the experimental outcome of our current research. We conclude our research with a discussion of the limitations and future prospects of our research.

\section{Algorithm description and experimental setup}

In boosting, a relatively weak learning algorithm is repeatedly applied to a set of training data, with classifiers being produced at each iteration. These classifiers are finally combined into one composite classifier. Provided that the algorithm can produce classifiers that are at least slightly better than random guessing, boosting can significantly reduce the classification error rate [9].

With AdaBoost, which can be used to boost a wide range of learning algorithms, data instances are weighted after each iteration. AdaBoost is adaptive in that the weight of an instance is increased when the classification is incorrect. AdaBoost may be susceptible to noisy data, but is less susceptible to overfitting than most other learning algorithms. It can identify outliers which are usually the instances with the greatest weight [10].

AdaBoost.M1 and AdaBoost.M2 were developed by Schapire and Freund from their AdaBoost algorithm. For binary classification problems the two versions are equivalent, differing only in the way they handle problems with more than two classes [9].

AdaBoost.M1 has access to a learning algorithm (which the developers generically title WeakLearn) which it calls repeatedly with distributions over the training set. WeakLearn calculates a hypothesis, or classifier, that attempts to correctly classify all instances of the test data. As described previously, examples that are incorrectly classified are given 
greater weighting for the next pass. Finally, the boost algorithm combines all the hypotheses into one final hypothesis [9].

We chose four classifiers, namely J48, DecisionStump, NaïveBayes and PART as a base classifier for the AdaBoostM1 algorithm. All these classifiers are available in the WEKA [11] implementation. WEKA is a Java based machine learning tools. The DecisionStump classifier is a default setting with AdaBoostM1 in WEKA. The following section provides a brief explanation about the base classifiers.

J48: J4.8 is a supervised learning algorithm which induces a decision tree. It was developed by the developers of the Weka package (please see below) and is based on the widely-used C4.5 algorithm developed by J.R.Quinlan [11]. A decision tree is a tool for carrying out classification of data instances input to it. Decision trees have production rules of the type IF THEN (IF feathers = 'yes' THEN Animal = 'bird'). Data are input firstly to the root node, which typically has a binary output, leading to two edges ('branches'). Which edge is followed depends on the answer to the condition posed in the node. Each edge may lead to a subsequent node with further outputs. Finally, the choices lead to a 'leaf' node which definitively classifies the instance into one class or another with no further choices [12].

DecisionStump: A DecisionStump is a simplified Decision Tree, having just one level. Usually a weak classifier, decision stumps are commonly used with a boosting algorithm to markedly increase classification accuracy. Interestingly, missing values are simply treated as a third class [11].

NaïveBayes: Naïve Bayes is based on the wellknown Bayes Theorem. It is termed 'naïve' because it assumes that attributes of the training set are conditionally independent and that the prediction procedure is not influenced by any hidden or latent attributes. It works by calculating the maximum posterior probability of each class $[13,14]$.

PART: Part is developed from the C4.5 and RIPPER algorithms and is a partial decision tree algorithm. However, unlike C4.5 and RIPPER, PART does not have to perform global optimization in order to generate rules [14].

We fed all these base algorithms with AdaBoostM1 to classify a wide range of problems. First, we fed each classifier one-by-one with AdaBoostM1 and kept a record of the classification performance for the 113 problems. We selected all data sets from two different data repositories $[15,16]$. All classification problems descriptions are available in Appendix I. We chose ten- fold cross validation over the experiment. Then we collected the descriptive statistical information about each of the 113 classification problems. The list of descriptive statistics is follows:

$\begin{array}{ll}\text { Statistical Name } & \text { Symbolic Name } \\ \text { Geometric mean } & \text { geomean } \\ \text { harmonic mean } & \text { harmmean } \\ \text { statistical mean } & \text { sm } \\ \text { median } & \text { me } \\ \text { trim mean, } & \text { trimmean } \\ \text { inter quartile range } & \text { iqr } \\ \text { mad } & \text { ma } \\ \text { range } & r \\ \text { standard deviation } & \text { std } \\ \text { variance } & v \\ \text { prctile } & p \\ \text { chi-square cumulative distribution } & \text { chiscdf } \\ \text { normal cumulative distribution } & n o r m c d f \\ \text { skewness } & s \\ \text { kurtosis } & k \\ \text { correlation coefficient } & c c \\ \text { Z-score } & z\end{array}$

The explanations of these descriptive statistical terms are available in any statistical text book. Moreover one can find the implementation in the Statistical Toolbox in Matlab [17].

We constructed a data matrix with these statistical algorithms and the name of the best algorithm performance. Then we employed the C5.0 [18] algorithm to generate the rules. These rules have been considered to select a unique classifier for AdaBoostM1 algorithms to classify any problem with better accuracy and faster computation.

\section{Experimental results}

We observed from the experiment that the J48 classifier is the best choice for the AdaBoostM1 algorithm and it shows the highest percentage of average accuracy for the 113 problems. However, in terms of computational complexity DecisionStump is the best choice among the four classifiers.

The rules were generated using the C5.0 decision tree algorithm to select a unique classifier for the AdaBoostM1 algorithm. C5.0 has two parameters, pruning confidence (c) and minimum cases (m). Pruning confidence affects error estimation and therefore how severely the tree may be pruned; a smaller value of $\mathrm{c}$ enables more pruning and a higher value less pruning. Minimum cases affect how the tree fits the data; a higher value of $\mathrm{m}$ allows more prepruning. The value of $\mathrm{m}$ should be at least two for 
every node in the tree [14]. We tuned both parameters to produce the best rule. The generated rules were verified by ten-fold cross validation and the percentage of accuracy is summarized with the rules. These rules are as follows:

\subsubsection{Rules for J48 Classifier}

Rule: IF $Z>71.932$ OR harmmean $<=36.87$ and $s>1.6085$ OR harmmean $<=36.87$ and $p>$ 18.2 OR trimmean $>57.731$ THEN select J48 Classifier for AdaBoostM1 Algorithm.

$$
\text { Rule Accuracy }=85.72 \%
$$

\subsubsection{Rules for DecisionStump Classifier}

Rule: IF $s<=1.6085$ and $z>40.276$ OR geomean $<=0.4649$ and trimmean $>0.88472$ and $s$ $<=1.6085$ OR geomean $>117.06$ OR $m a<=$ 0.96388 and $k<=5.2902$ OR trimmean $<=57.731$ and $s<=1.6085$ THEN select DecisionStump Classifier for AdaBoostM1 Algorithm.

Rule Accuracy $=81.24 \%$

\subsubsection{Rules for NB Classifier}

Rule: IF trimmean $<=57.731$ and normcdf $<=$ 0.84223 and $s>0.77518$ and $s<=1.736$ and $Z>$ 18.205 OR geomean $<=8.97$ and iqr $<=2.2064$ and normcdf $>0.84223$ and $c c>0.17111$ OR trimmean $<=57.731$ and $s<=1.736$ THEN select NB Classifier for AdaBoostM1 Algorithm.

Rule Accuracy $=80 \%$

\subsubsection{Rules for PART Classifier}

Rule: IF trimmean $>81.997$ and trimmean $<=$ 98.664 OR harmmean $>5.0671$ and trimmean $<=$ 98.664 and $i q r>14.778$ and $s<=13.596$ OR geomean $<=117.06$ and trimmean $>137.04$ OR $r$ $<=0.652$ and $s>1.7405 \mathrm{OR}$ iqr $<=14.778$ and normcdf $>0.85796$ THEN select PART Classifier for AdaBoostM1 Algorithm.

Rule Accuracy $=70.59 \%$

\section{Conclusions}

This research contributes a new approach to selecting a unique classifier for the AdaBoostM1 algorithm. A rule based approach has been introduced for the unique classifier selection. These rules are generated based on described statistical information of 113 classification problems. All generated rules showed higher accuracy during the ten-fold cross validation except for the PART algorithm. The PART algorithm showed the best classification performance for only a few data sets. This performance could be increased by considering more classification problems. We have planned to extend our research using more problems from different domains with a variety of classifiers.

\section{References}

[1] R. E. Schapire, "The strength of weak learnability," Machine Learning, vol. 5, no. 2 pp 197-227, 1990

[2] Y. Freund, "Boosting a weak learning algorithm by majority," Information and Computation, vol.121 no. 2 pp 256-285, 1995.

[3] A. Grove and D. Schuurmans, "Boosting in the limit: Maximizing the margin of learned ensembles," in Proceedings of the Fifteenth National Conference on Artifical Intelligence 1998, pp. 692-699.

[4] G. Ratsch, T. Onoda and K. Muller, "Regularizing AdaBoost," Advances in Neutral Information Processing Systems 11. 1999.

[5] G. Ratsch, T. Onoda and K. Muller, "Soft Margins for AdaBoost," Machine Learning, 42, pp287-320, 2000.

[6] G. Lebanon and J. Lafferty, "Boosting and maximum likelihood for exponential models," Neural Information Processing Systems (NIPS). 2001.

[7] R. Jin, Y. Liu, L. Si, J. Carbonell and A. Hauptmann, “A New Boosting Algorithm Using Input-Dependent Regularizer," in Proceedings of Twentieth International Conference on Machine Learning (ICML'03) 2003.

[8] Y. Freund and R. E. Schapire, "A decision-theoretic generalization of online learning and an application to boosting", Unpublished manuscript available electronically (on our web pages, or by email request). An extended abstract appeared in Computational Learning Theory: Second European Conference, EuroCOLT '95, pp 23-37, Springer-Verlag, 1995.

[9] Y. Freund and R.E. Schapire, "Experiments with a new boosting algorithm," In the Proceeding of International Conference on Machine Learning, pp 148-156, 1996.

[10] P. Radeva, Theoretical analysis of AdaBoost, online, accessed 15 February 2007. Available at:

http://iua-share.upf.es/wikis/seminaris/images/5/59/

Adaboost.pdf

[11] I.H. Witten and E. Frank, Data Mining: Practical Machine Learning Tools and Techniques, Morgan Kaufmann, 2005.

[12] P. Tan, M. Steinbach and V. Kumar, Introduction to Data Mining, Addison-Wesley, 2006.

[13] G.H. John and P. Langley, "Estimating continuous distributions in Bayesian classifiers," in Proceedings of the Eleventh Conference on Uncertainty in Artificial Intelligence, Morgan Kaufmann, San Mateo, CA, pp. 338345, 1995.

[14] S. Ali and K.A. Smith, "On Learning Algorithm Selection for Classification," Applied Soft Computing, Elsevier Science, vol.6, no. 2, pp.119-138, January 2006.

[15] C. Blake and C.J. Merz, UCI Repository of machine learning databases, University of California, Irvine, CA, 2002. http://www.ics.uci.edu/mlearn/ mlrepository.html. 
[16] T. S. Lim, Knowledge Discovery Central datasets, 2002, http://www.kdcentral.com/.

[17] Statistics Toolbox User's Guide, version 3, The MathWorks Inc., USA, 2001. URL: http://www. mathworks.com.

[18] See 4: http://www.rulequest.com/see5-info.html, Accessed: 02 February 2007.

Appendix I: Datasets description.

\begin{tabular}{|c|c|c|c|c|}
\hline $\begin{array}{l}\# \\
\text { Data } \\
\text { set }\end{array}$ & Data set name & $\begin{array}{l}\text { \# } \\
\text { Instances }\end{array}$ & $\begin{array}{l}\text { \# } \\
\text { Attributes }\end{array}$ & $\begin{array}{l}\# \\
\text { Class }\end{array}$ \\
\hline 1 & abalone & 1253 & 9 & 3 \\
\hline 2 & adp & 1351 & 12 & 3 \\
\hline 3 & adult + stret & 20 & 5 & 2 \\
\hline 4 & adult-stret & 20 & 5 & 2 \\
\hline 5 & allbp & 840 & 7 & 3 \\
\hline 6 & ann1 & 1131 & 7 & 3 \\
\hline 7 & ann2 & 1028 & 7 & 3 \\
\hline 8 & aph & 909 & 19 & 2 \\
\hline 9 & art & 1051 & 13 & 2 \\
\hline 10 & australian & 690 & 15 & 2 \\
\hline 11 & balance-sca & 625 & 5 & 3 \\
\hline 12 & bcw & 699 & 10 & 2 \\
\hline 13 & bcw_noise & 683 & 19 & 2 \\
\hline 14 & bld & 345 & 7 & 2 \\
\hline 15 & bld noise & 345 & 16 & 2 \\
\hline 16 & bos & 910 & 14 & 3 \\
\hline 17 & bos_noise & 506 & 26 & 2 \\
\hline 18 & breast-canc & 286 & 7 & 2 \\
\hline 19 & breast-canc & 699 & 10 & 2 \\
\hline 20 & bupa & 345 & 7 & 2 \\
\hline 21 & $\mathrm{c}$ & 1500 & 16 & 2 \\
\hline 22 & cleveland-heart & 303 & 14 & 5 \\
\hline 23 & $\mathrm{cmc}$ & 1473 & 10 & 3 \\
\hline 24 & crx & 490 & 16 & 2 \\
\hline 25 & dar & 1378 & 10 & 5 \\
\hline 26 & dhp & 1500 & 8 & 2 \\
\hline 27 & DNA-n & 1275 & 61 & 3 \\
\hline 28 & dna & 2000 & 61 & 3 \\
\hline 29 & dna_noise & 2000 & 81 & 3 \\
\hline 30 & $\mathrm{dph}$ & 590 & 11 & 2 \\
\hline 31 & echocardiogram & 131 & 8 & 2 \\
\hline 32 & flare & 1389 & 11 & 2 \\
\hline 33 & german & 1000 & 25 & 2 \\
\hline 34 & glass & 214 & 10 & 6 \\
\hline 35 & h-d & 303 & 14 & 2 \\
\hline 36 & hayes-roth & 132 & 6 & 3 \\
\hline 37 & hea & 270 & 14 & 2 \\
\hline 38 & hea_noise & 270 & 21 & 2 \\
\hline 39 & heart & 270 & 14 & 2 \\
\hline 40 & hepatitis & 155 & 20 & 2 \\
\hline 41 & horse-23 & 368 & 23 & 2 \\
\hline 42 & horse-colic & 368 & 28 & 2 \\
\hline 43 & house-votes-84 & 435 & 17 & 2 \\
\hline
\end{tabular}

\begin{tabular}{|c|c|c|c|c|}
\hline 44 & hyp & 2847 & 16 & 2 \\
\hline 45 & hypothyroid & 1265 & 26 & 2 \\
\hline 46 & iris & 150 & 5 & 3 \\
\hline 47 & khan & 1063 & 6 & 2 \\
\hline 48 & kr-vs-kp & 1279 & 37 & 2 \\
\hline 49 & labor-neg & 40 & 17 & 2 \\
\hline 50 & led-noise & 1047 & 10 & 10 \\
\hline 51 & lenses & 24 & 6 & 3 \\
\hline 52 & letter-a & 1334 & 17 & 2 \\
\hline 53 & lung-cancer & 32 & 57 & 2 \\
\hline 54 & lymphography & 148 & 19 & 8 \\
\hline 55 & mha & 1269 & 9 & 4 \\
\hline 56 & monk1 & 556 & 7 & 2 \\
\hline 57 & monk2 & 601 & 7 & 2 \\
\hline 58 & monk3 & 554 & 7 & 2 \\
\hline 59 & mushroom & 1137 & 12 & 2 \\
\hline 60 & nettalk_str & 1141 & 8 & 5 \\
\hline 61 & page-blocks & 1149 & 11 & 5 \\
\hline 62 & pendigits- 8 & 1399 & 17 & 2 \\
\hline 63 & pha & 1070 & 10 & 5 \\
\hline 64 & phm & 1351 & 12 & 3 \\
\hline 65 & phn & 1500 & 10 & 2 \\
\hline 66 & pid & 532 & 8 & 2 \\
\hline 67 & Pima & 768 & 9 & 2 \\
\hline 68 & poh & 527 & 12 & 2 \\
\hline 69 & post-operative & 90 & 9 & 3 \\
\hline 70 & primary-tum & 339 & 18 & 2 \\
\hline 71 & pro & 1257 & 13 & 2 \\
\hline 72 & promoter & 106 & 58 & 2 \\
\hline 73 & pvro & 590 & 19 & 2 \\
\hline 74 & rph & 1093 & 9 & 2 \\
\hline 75 & satimage & 1351 & 11 & 6 \\
\hline 76 & $\begin{array}{l}\text { shuttle-landing } \\
\text { control }\end{array}$ & 15 & 7 & 2 \\
\hline 77 & sick-euthyroid & 1582 & 16 & 2 \\
\hline 78 & sma & 409 & 8 & 4 \\
\hline 79 & smo & 1855 & 9 & 2 \\
\hline 80 & smo_noise & 1855 & 16 & 2 \\
\hline 81 & sonar & 208 & 61 & 2 \\
\hline 82 & splice & 1589 & 61 & 3 \\
\hline 83 & $t$ _series & 62 & 3 & 2 \\
\hline 84 & tae & 151 & 6 & 3 \\
\hline 85 & tae_noise & 151 & 11 & 2 \\
\hline 86 & thy & 1887 & 22 & 3 \\
\hline 87 & thynoise & 1132 & 11 & 3 \\
\hline 88 & tic-tac-toe & 958 & 10 & 2 \\
\hline 89 & titanic & 2201 & 4 & 2 \\
\hline 90 & tmris & 100 & 4 & 2 \\
\hline 91 & tqr & 1107 & 12 & 2 \\
\hline 92 & $\begin{array}{l}\text { trains- } \\
\text { transformed }\end{array}$ & 10 & 17 & 2 \\
\hline 93 & va-heart & 200 & 9 & 4 \\
\hline 94 & veh & 846 & 19 & 4 \\
\hline 95 & veh_noise & 761 & 31 & 4 \\
\hline 96 & vehicle & 658 & 20 & 0 \\
\hline
\end{tabular}




\begin{tabular}{|r|l|r|r|r||}
\hline 97 & votes_noise & 391 & 31 & 2 \\
\hline 98 & waveform & 5000 & 22 & 2 \\
\hline 99 & waveform_noise & 5000 & 41 & 2 \\
\hline 100 & wdbc & 569 & 31 & 2 \\
\hline 101 & wine & 178 & 14 & 3 \\
\hline 102 & wpbc & 199 & 34 & 2 \\
\hline 103 & xaa & 94 & 19 & 4 \\
\hline 104 & xab & 94 & 19 & 4 \\
\hline 105 & xac & 94 & 19 & 4 \\
\hline 106 & xad & 94 & 19 & 4 \\
\hline 107 & xae & 94 & 19 & 4 \\
\hline 108 & xaf & 94 & 19 & 4 \\
\hline 109 & xag & 94 & 19 & 4 \\
\hline 110 & xah & 94 & 19 & 4 \\
\hline 111 & xai & 1601 & 10 & 2 \\
\hline 112 & yha & & 17 & 7 \\
\hline 113 & zoo & & & \\
\hline
\end{tabular}

